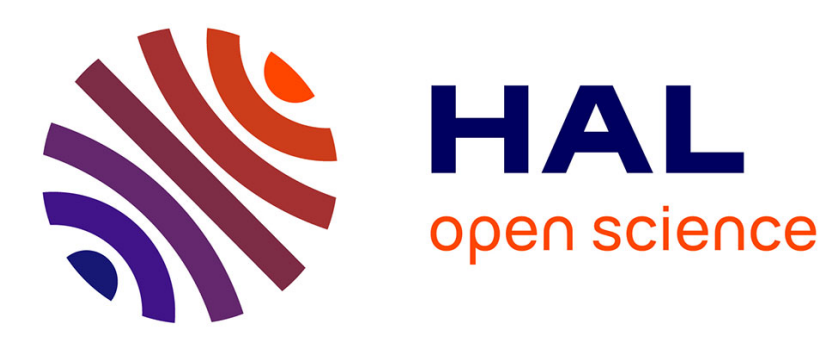

\title{
Colour space influence for vegetation image classification Application to Caribbean forest and agriculture
}

\author{
Mohamed Abadi, Enguerran Grandchamp
}

\section{To cite this version:}

Mohamed Abadi, Enguerran Grandchamp. Colour space influence for vegetation image classification Application to Caribbean forest and agriculture. SPIE Remote Sensing, Sep 2008, Cardiff, United Kingdom. 10.1117/12.799886 . hal-00602277

\section{HAL Id: hal-00602277 \\ https://hal.science/hal-00602277}

Submitted on 2 Mar 2020

HAL is a multi-disciplinary open access archive for the deposit and dissemination of scientific research documents, whether they are published or not. The documents may come from teaching and research institutions in France or abroad, or from public or private research centers.
L'archive ouverte pluridisciplinaire HAL, est destinée au dépôt et à la diffusion de documents scientifiques de niveau recherche, publiés ou non, émanant des établissements d'enseignement et de recherche français ou étrangers, des laboratoires publics ou privés. 


\title{
Colour space influence for vegetation image classification Application to Caribbean forest and agriculture
}

\author{
M. Abadi ${ }^{\mathrm{a}}$, E. Grandchamp ${ }^{\mathrm{a}}$ \\ ${ }^{a}$ Université des Antilles et de la Guyane UFR-SEN Laboratoire GRIMAAG Campus de Fouillole \\ 97159 Guadeloupe France
}

\begin{abstract}
This paper deals with a comparison of different colour space in order to improve high resolution images classification. The background of this study is the measure of the agriculture impact on the environment in islander context. Biodiversity is particularly sensitive and relevant in such areas and the follow-up of the forest front is a way to ensure its preservation. Very high resolution satellite images are used such as QuickBird and IKONOS scenes.

In order to segment the images into forest and agriculture areas, we characterize both ground covers with colour and texture features. A classical unsupervised classifier is then used to obtain labelled areas. As features are computed on coloured images, we can wonder if the colour space choice is relevant. This study has been made considering more than fourteen colour spaces (RGB, YUV, Lab, YIQ, YC $C_{r} C_{s}, X Y Z, C M Y, L M S, H S L, K L T, I H S, I_{1} I_{2} I_{3}, H S V$, HSI, etc.) and shows the visual and quantitative superiority of IHS on all others. For conciseness reasons, results only show $R G B, I_{1} I_{2} I_{3}$ and IHS colour spaces.
\end{abstract}

Keywords: Classification, colour space, texture features, high resolution images, Gabor filters, Hu moments

\section{Introduction}

During last years, the use of very high resolution satellite images has considerably increased. More specialy for geographical information system which was essentially, in 1990, an administration activity. The main goal of our study is to develop tools for natural forest resources supervision. In this way, we propose a classification tool which is able to separate forest and agriculture areas over Caribbean islands. Following the forest boundaries will help the authority on charge of forest preservation to control and prevent human effect on nature.

With the arrival of very high satellite images (less than 5 meters per pixel) this field has been completed with new available problematic. Table 1.1 show the spatial resolution for different satellites and for panchromatic (PAN) and Multispectral (MS) band.

\begin{tabular}{|l|c|c|c|}
\hline & IKONOS & QUICKBIRD & SPOT5 \\
\hline PAN & 1 & 0,61 & 2,5 \\
\hline MS & 4 & 2,44 & 5 \\
\hline
\end{tabular}

Table 1.1: satellite image resolution in meter per pixel

But this accurate spatial resolution has some drawbacks. Indeed, homogenous areas obtained with coarse resolutions are now composed of complex structures with sub areas having different textures.

Different approaches could be used to analyze such images. The texture approach [3-4] which try to characterize the chaotic aspect of the image. This could be done with a structural approach base on the shape and using tools like Fourier analysis [7], wavelet analysis [8] or Gabor filters [9]. The colour approach [10] which require the definition of a colour space. Few studies are based on colour space to analyze satellites images and still less which combined colour and texture approaches. Our study presents an approach in this way. 
Different method could be found to characterize textures (statistical [11], geometrical [12], frequential [13] and methods based on models [14]). We adopt in this paper the following methods: a structural approach based on Hu moments[16] computed with geometrical moments [17] et a signal processing approach with Gabor filters [18].

Many studies [15] have been made in order to define colorimetric functions $R(\lambda), G(\lambda)$ and $B(\lambda)$ and many more to define the colour spaces to represent the functions (XYZ, L* $\left.\mathrm{u}^{*} \mathrm{v}^{*}, \mathrm{~L}^{*} \mathrm{a}^{*} \mathrm{~b}^{*}, \mathrm{HSV}, \mathrm{I}_{1} \mathrm{I}_{2} \mathrm{I}_{3}, \ldots\right)$. In this paper we use three color spaces RVB, HSI et $\mathbf{I}_{1} \mathbf{I}_{2} \mathbf{I}_{3}$.

This paper is organized as follow: section 2 describes the adopted approach. Sections 3, 4 and 5 respectively deal with colour spaces, pan sharpening techniques and feature extraction. Images used for application are presented in section 6 . Experimental results with synthetic and real images are presented in section 7. A comparative study is realized in section 8. Finally section 9 gives some conclusion and perspectives to our work.

\section{Adopted approach}

In this section we describe the different steps of our approach in order to separate agricultural and forest areas.

The algorithm (Figure 2.1) includes a image fusion procedure detailed later to improve the spatial resolution of the multispectral images. The features are computed using methods described in section 5. Finally an unsupervised classification procedure based on K-means algorithm is used.

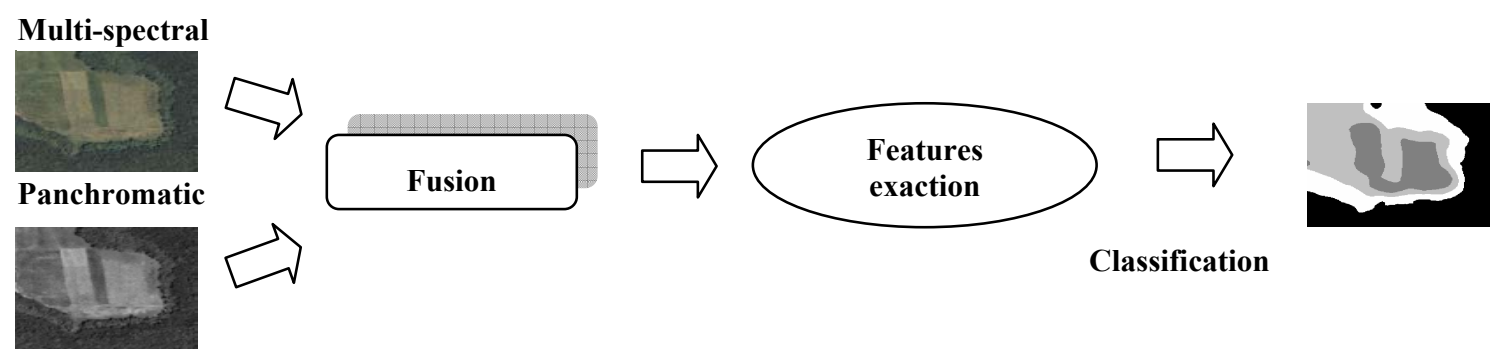

Figure 2.1: Adopted algorithm

\section{Color space}

The efficiency of a classification method is known to depend on the choice of the color space used to represent the image. Many studies have been realized to give a way to select the appropriate color space for a specific application. In the scope of this paper we investigate three color spaces: RGB, $\mathrm{I}_{1} \mathrm{I}_{2} \mathrm{I}_{3}$, HSI.

\subsection{RGB colour space}

RGB is defined with three based colours ( $\mathrm{R}$ : red, G : green and B : blue). This is the base of colour images acquisition, it is the most used colour space in image analysis but it's not the most adapted in many cases. In fact, the main drawback of this colour space for natural images is the correlation between the components and the non intuitive relation with human vision.

\section{2 $\mathbf{I}_{1} \mathbf{I}_{2} \mathbf{I}_{3}$ colour space}

Introduce by Otha and al. [20] for segmentation and image analysis with the aim to obtain uncorrelated components, the authors have proved it's efficiency. The resulting colour space is comparable to an PCA (Principal Component Analysis) with less time consuming. This colour space is defined with the following expressions. 


$$
\begin{aligned}
& I_{1}=\frac{R+v+B}{3} \\
& I_{2}=\frac{R-B}{2} \\
& I_{3}=\frac{2 V-R-B}{4}
\end{aligned}
$$

We remark that $\mathbf{I}_{\mathbf{1}}$ represent the luminosity and $\mathbf{I}_{\mathbf{2}}$ et $\mathbf{I}_{\mathbf{3}}$ the chromacity. This repsentation increased the importance of chromacity in order to be closer to human vision.

\subsection{HSI colour space}

Introduce to intuitively represent the colour as human vision done. It separates the intensity information from the chromacity information. Three components are defined: the Hue which represent the pure colour (i.e. wave length) and allow to give information about dominating colour, the saturation which inform about the white ratio of the colour and the intensity which measure the amount of light of the colour.

We adopted for this study the transformation defined by Sangwine and al. [19] to pass from RGB to HSI :

$$
\begin{aligned}
& I=\frac{R+V+B}{3} \\
& S=1-\frac{\min (R, V, B)}{I} \\
& H=\left\{\begin{array}{lll}
\frac{V-B}{I-\min (R, V, B)} & \text { si } & V=R \\
2+\frac{B-R}{I-\min (R, V, B)} & \text { si } & V=G \\
4+\frac{R-V}{I-\min (R, V, B)} & \text { si } & V=B
\end{array}\right.
\end{aligned}
$$

\section{Pansharpening technics}

This is an image fusion method to improve the spatial resolution of the multi-spectral images. Many treatment applied to satellite images use such technics [21-23]. We used in this paper the pansharpening technic based on the HSI transform described in Figure 4.1.

\section{Multi-spectral image}

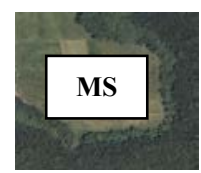

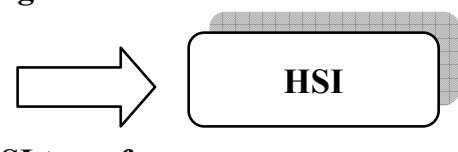

HSI transform
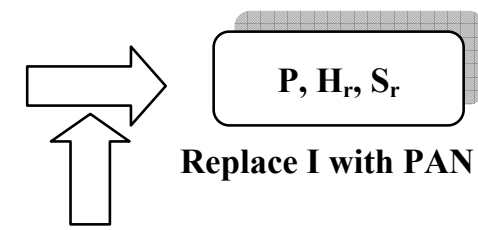

Replace I with PAN

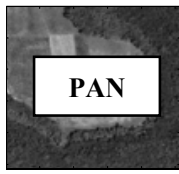

Figure 4.1 : Pansharpening technics based on HSI transform

Over-sampling of $\mathbf{H}$

Panchromatic image

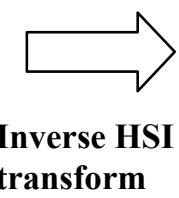

transform

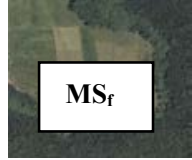

Fused Multispectral images and $S$ to fit 


\section{Features extraction}

Features extraction is the most important step to ensure a good classification quality. For this reason the selected features must be discriminant for the researched classes. In this way we use both colour and texture features.

\subsection{Hu moments}

We use the classical formulation of bi-dimension moments to describe the main aspect of the textures present in the image. This statistical properties are given with the following generic equation:

$$
m_{p, q}=\sum_{x \in W} \sum_{y \in W} x^{p} y^{q} I(x, y), \quad \forall(p, q) \in N^{2}
$$

Where the moment order is $p+q, I(x, y)$ is the image at $x, y$ coordinates and $W$ is a window centere onthe current pixel coordinates $(x, y)$.

After normalisation we obtained:

$\eta_{p, q}=\sum_{x \in W} \sum_{y \in W}(x-\bar{x})^{p}(y-\bar{y})^{q} I(x, y)$

where $\bar{x}=\frac{m_{10}}{m_{00}}$ and $\bar{y}=\frac{m_{01}}{m_{00}}$ are the gravity points. This new expression is independent from scale.

From this normalized geometrical moments, Hu [16] introduces seven notions which are invariant with translation, rotation or scaling. This notions are combination of the following equation:

$\mu_{p, q}=\frac{\eta_{p, q}}{\eta_{00}^{1+\frac{(p+q)}{2}}} \quad \forall(p+q) \geq 2$ et $(p, q) \in N^{2}$

The expressions are given by:

$$
\left\{\begin{aligned}
M_{1} & =\mu_{20}+\mu_{02} \\
M_{2} & =\left(\mu_{20}-\mu_{02}\right)^{2}+4 \mu_{11}^{2} \\
M_{3} & =\left(\mu_{30}-3 \mu_{12}\right)^{2}+\left(3 \mu_{21}-\mu_{03}\right)^{2} \\
M_{4} & =\left(\mu_{30}+\mu_{12}\right)^{2}+\left(\mu_{21}+\mu_{03}\right)^{2} \\
M_{5} & =\left(\mu_{30}-3 \mu_{12}\right)\left(\mu_{30}-\mu_{12}\right)\left[\left(\mu_{30}+\mu_{12}\right)^{2}\right. \\
& \left.-3\left(\mu_{21}+\mu_{03}\right)^{2}\right]+\left(3 \mu_{12}-\mu_{03}\right)\left(\mu_{21}+\mu_{03}\right) \\
& *\left[3\left(\mu_{30}+\mu_{12}\right)^{2}-\left(\mu_{21}+\mu_{03}\right)^{2}\right] \\
M_{6} & =\left(\mu_{20}-\mu_{02}\right)\left[\left(\mu_{30}+\mu_{12}\right)^{2}-\left(\mu_{21}+\mu_{03}\right)^{2}\right] \\
& +4 \mu_{11}\left(\mu_{30}-\mu_{12}\right)\left(\mu_{03}+\mu_{21}\right) \\
M_{7} & =\left(3 \mu_{21}-\mu_{03}\right)\left(\mu_{30}+\mu_{12}\right)\left[\left(\mu_{30}+\mu_{12}\right)^{2}\right. \\
& \left.-3\left(\mu_{21}+\mu_{03}\right)^{2}\right]-\left(\mu_{30}-3 \mu_{21}\right)\left(\mu_{21}+\mu_{03}\right) \\
& *\left[3\left(\mu_{30}+\mu_{12}\right)^{2}-\left(\mu_{12}+\mu_{03}\right)^{2}\right]
\end{aligned}\right.
$$


These moments give discriminant features to describe and classify textures but they are time consuming and noise sensitive.

After moment computation over each image component $(i=1,2,3)$ a moment descriptor is defined as follow:

$$
H_{i}=\left\lfloor M_{j}\right\rfloor \quad \text { avec } \quad j=1, \cdots, 7
$$

It will be used for classification procedure.

\subsection{Gabor filters}

This filters results from a multiplication in the space field of a gaussian function $g$ with a complex sinusoid function oriented with a $\theta=\frac{r \pi}{R}(r=0,1,2, \cdots)$ angle with $x$ axis. Their efficiency to recognize and discriminate textures has been proved [24]. In the space field, for a sinusoid having frequencies $\mu$ and $v$ along $x$ and $y$ axis the 2D Gabor filters are:

$$
\begin{aligned}
& h(x, y)=\frac{1}{a^{s}} g\left(x^{\prime}, y^{\prime}\right) \cdot \exp \left[2 \pi i\left(\mu x^{\prime}+v y^{\prime}\right)\right] \\
& \text { with } \\
& \left(x^{\prime}, y^{\prime}\right)=\frac{1}{a^{s}}(x \cos \theta+y \sin \theta,-x \sin \theta+y \cos \theta), \\
& g(x, y)=\frac{1}{2 \pi \sigma_{x} \sigma_{y}} \exp \left\{-\frac{1}{2}\left[\frac{x}{\sigma_{x}}\right]^{2}+\left[\frac{y}{\sigma_{y}}\right]^{2}\right\},
\end{aligned}
$$

where $\sigma_{x}$ (resp. $\sigma_{y}$ ) is the standard deviation of the Gaussian function $g$ along $x$ axis (resp. $y$ axis). The index $s=0,1, \cdots S-1, R$ and $S$ respectively represent the scale, the number of orientation and the filters scale. The coefficient computation is based on the equations defined by Manjunath and al. [24].

With this formulation Gabor filters allow to cover the main part of frequency space and the projection of a texture on this filters is representative

In the following, the images $I_{i}(x, y) \quad(i=1,2,3$ is the colour component) are convoluted by the $R \times S$ filters. The mean and standard deviation of this convolutions are computed to give a feature vector $G_{i}$.

$G_{i}=\left[m_{11}, \sigma_{11}, \cdots, m_{R S}, \sigma_{R S}\right]$

This vector will be used for the classification step[25].

\section{Data used}

In this paper we first apply our approach on two synthesis images. The first (figure 6.1 (a)) is composed by four texture samples randomly choose from VisTex data base [1]. The second one is built from IKONOS images extracts having a $1 \mathrm{~m}$ resolution (figure 6.1 (b)) after pansharpening application (section 4).

Then real images representing Guadeloupe Island in the Caribbean see are employed to validate our approach (figure 6.2 (a) et (b)). These images are composed of agricultural and forest areas. 


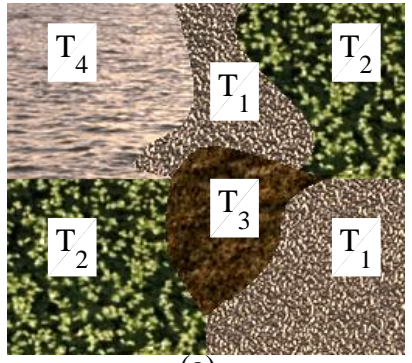

(a)

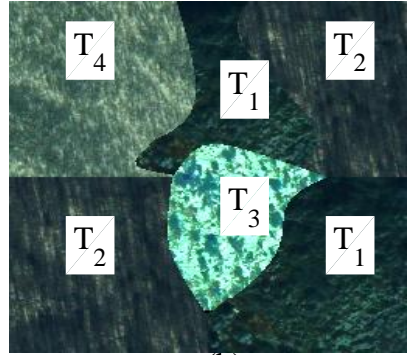

(b)

Figure 6.1: synthesis images built from VisTex database (a) and IKONOS extracts (b).

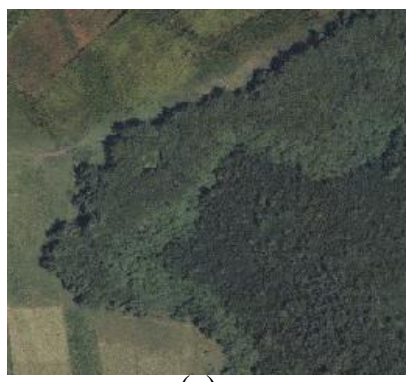

(a)

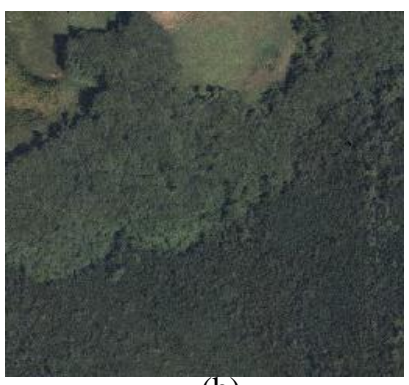

(b)

Figure 6.2: real images extracted from IKONOS scene

For the Hu moment method we use the seven moments for each colour space, so the feature vector size is 21 .

For the Gabor filters method the computation is made for six $(R=6)$, four scales $(S=4)$ and each colour space, so the feature vector size is $72(3 \times 6 \times 4)$.

\section{Experimental results}

We present in this section the results obtained with previous features computed on the RVB, $\mathbf{I}_{1} \mathbf{I}_{2} \mathbf{I}_{3}$ and $\mathbf{H S I}$ colour spaces. A k-means algorithm is used as classification algorithm.

The figure 7.1 shows that classification obtained with RVB is similar to the one obtained with $\mathbf{I}_{1} \mathbf{I}_{2} \mathbf{I}_{3}$. As the nature of $\mathbf{I}_{1} \mathbf{I}_{2} \mathbf{I}_{3}$ is similar to an PCA ACP [20], we can suppose that $\mathbf{I}_{\mathbf{2}}$ and $\mathbf{I}_{\mathbf{3}}$ components don't contain many information [20].

Moreover, the results obtained with $\mathbf{H S I}$ are better than the results obtained with $\mathbf{I}_{\mathbf{1}} \mathbf{I}_{2} \mathbf{I}_{\mathbf{3}}$. As $\mathbf{I}_{\mathbf{1}}=\mathbf{I}$ we can couclude that $\mathbf{H}$ and $\mathbf{S}$ components contains discriminant information. 
Résultats de la classification : figure 6.1 (a)
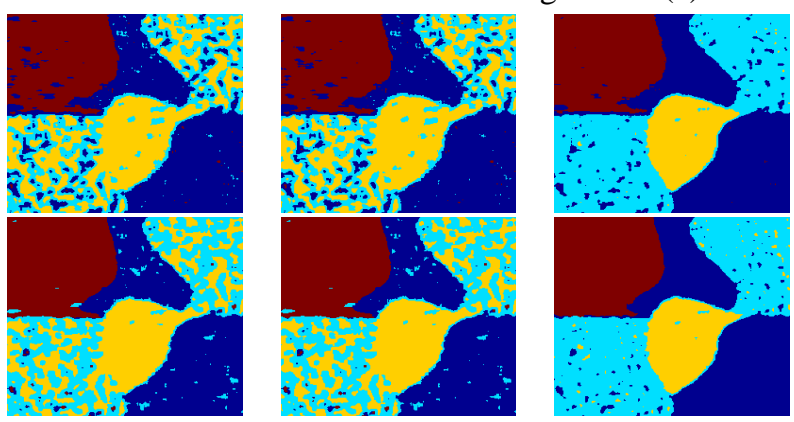

Résultats de la classification : figure 6.1 (b)

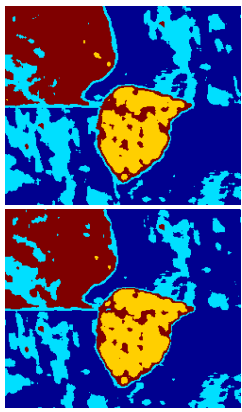

RVB

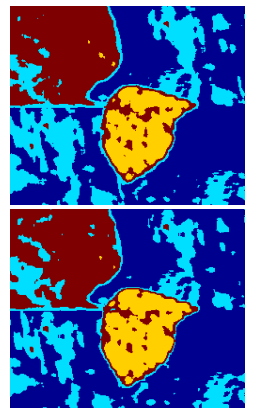

$\mathrm{I}_{1} \mathrm{I}_{2} \mathrm{I}_{3}$

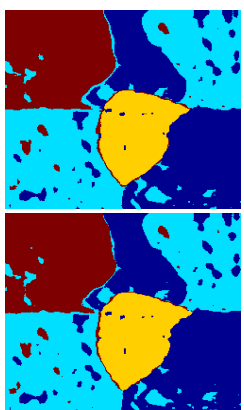

HSI

Figure 7.1: Classification results for synthesis images. Lines 1 and 3 using $\mathrm{Hu}$ moments. Lines 2 and 4 using Gabor filters.

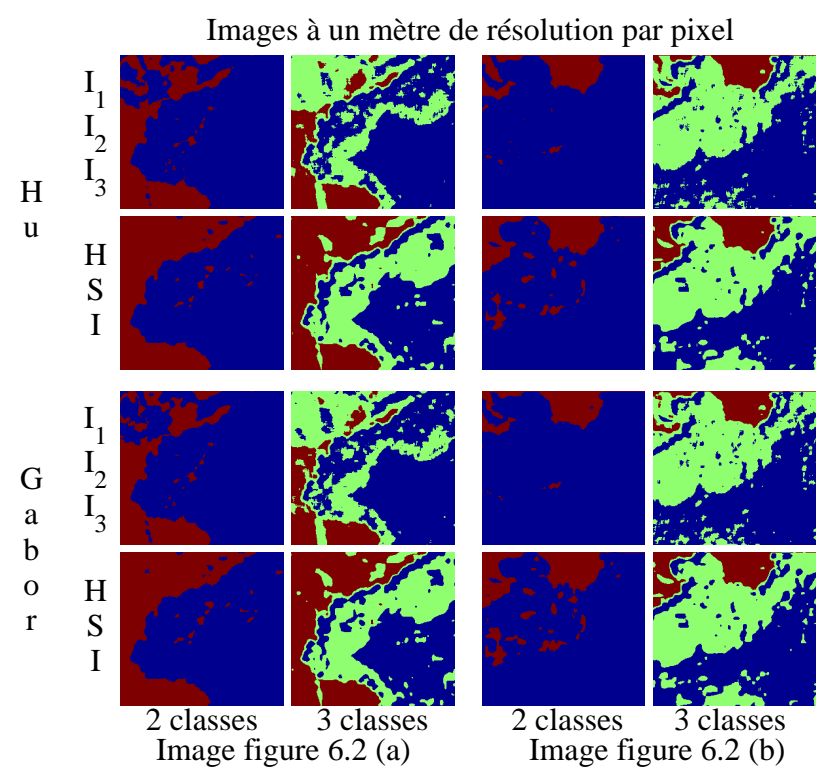

Figure 7.2. Classification results for real images.

Figure 7.2 confirms the previous observation for real scene extracts.

Moreover, the images are composed of two kinds of forests and one kind of agriculture (expert information). So we have done two classifications: one with two classes to separate agriculture and forest and one with three classes to separate the two kind of forest. The results are satisfying because we obtain in both cases ( 2 or 3 classes), with HSI colour space, the classes difined by the experts. 


\section{Comparative study}

This section will numerically support previous results by computing the good classification ratio for both synthesis images. So we could evaluate the contribution of each feature.

Information given by figure 8.1 show that good classification ratio are quite similar for $\mathbf{R V B}$ and $\mathbf{I}_{\mathbf{1}} \mathbf{I}_{\mathbf{2}} \mathbf{I}_{3}$ colour spaces. Meanwhile, we remark that this two spaces don't allow to distinguish classe 2 (texture $T_{2}$ ) which is not the case for HSI color space. In this case, the good classification ratio of this class is similar to the other one.

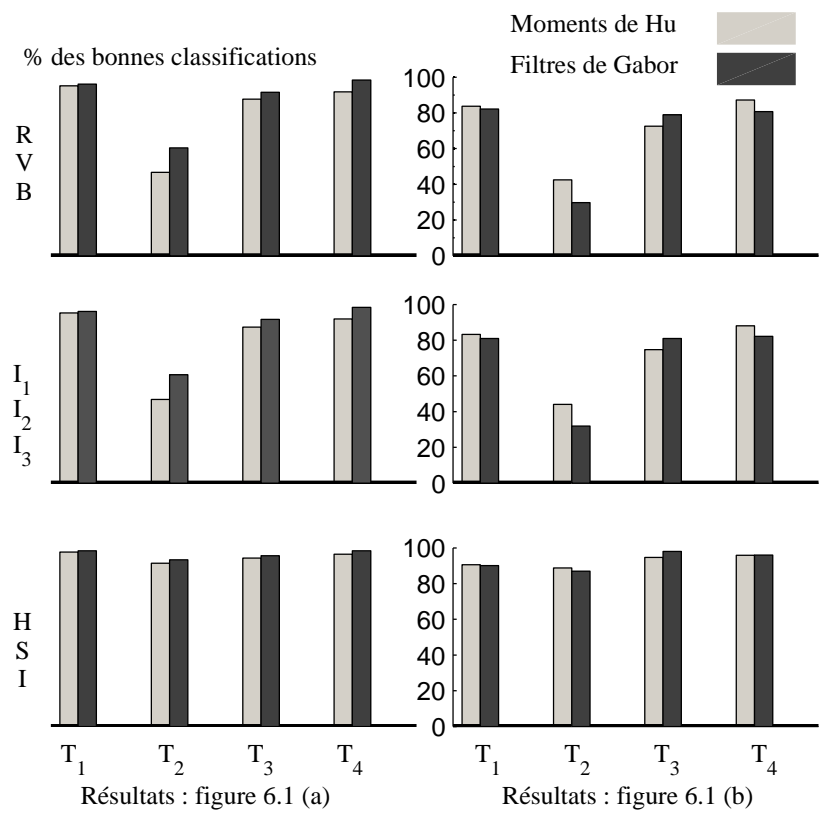

Figure 8.1: good classification ratio per class (texture).

The HSI colour space, for both synthesis images and for both methods (moments de Hu et les filtres de Gabor) allow to separate each texture.

The tables 8.1 and 8.2 shows a good classification ratio broadly higher in HSI case than in the other one.

\begin{tabular}{|c|c|c|c|}
\hline & RGB & $\mathrm{I}_{1} \mathrm{I}_{2} \mathrm{I}_{3}$ & HSI \\
\hline Hu moments & 0.7533 & 0.7535 & $\mathbf{0 . 9 4 6 4}$ \\
\hline Gabor filters & 0.8261 & 0.8272 & $\mathbf{0 . 9 6 1 3}$ \\
\hline
\end{tabular}

Table 8.1: global good classification ratio computed for image of figure 6.1 (a)

\begin{tabular}{|l|l|l|l|}
\hline & RGB & $\mathrm{I}_{1} \mathrm{I}_{2} \mathrm{I}_{3}$ & HSI \\
\hline Moments de $\mathrm{Hu}$ & 0.6763 & 0.6848 & $\mathbf{0 . 9 1 4 9}$ \\
\hline Filtres de Gabor & 0.6179 & 0.6277 & $\mathbf{0 . 9 1 0 9}$ \\
\hline
\end{tabular}

Table 8.2: global good classification ratio computed for image of figure 6.1 (b)

These results show the good performances of HSI colour space and the chosen textural features.

\section{Conclusion and perspectives}

The classification of textured colour images requires the selection of both discriminant features and colour space. We have presented in this way the Hu moments and the Gabor filters features which have proved their efficiency.

Both methods gives better results with HSI colour space than with $\mathbf{R V B}$ or $\mathbf{I}_{1} \mathbf{I}_{2} \mathbf{I}_{3}$ ones.

In this paper we show the importance of the colour space and its influence on classification results. This study support he idea of the non existance of a universal colour space. 
The two chromacity components $\mathbf{H}$ and $\mathbf{S}$ are more disciminant than $\mathbf{I}_{\mathbf{2}} \mathbf{I}_{\mathbf{3}}$. On conclusion, an approach which will optimize both color space and feature will allow saving computation time and is being develop.

\section{Acknowledgment}

The authors want to thanks the European institutions within the INTERREG III'b Caribbean Space for the financing of the CESAR project.

\section{References}

[1] MIT Media Lab Vision Texture-VisTex Database. http://www-white.media.mit.edu/vismod/imagery/VisionTexture/vistex.html.

[2] M. Abadi, S. Loumi, E. Grandchamp, and F. Alilat, Colour Image Texture Characterizing: Forest Classification's Island Guadeloupe by IKONOS Satellite, International Journal Remote Sensing, 2007 (soumis).

[3] R.M. Haralick, Statistical and structural approaches to texture. Proceedings of the IEEE, 67 (5), pp. 786-804, 1979.

[4] K.I. Laws, Rapid texture identification. In SPIE Image Processing for Missile Guidance, 238, pp. 376-380, 1980.

[10] J. Landré, and F. Truchetet, Content-based multirésolution indexing and retrieval of paleontology images, Proceeding of Storage and Retrieval for Media Databases, SPIE, vol. 4315, pp. 482-489, 2000.

[11] A. Al-Janobi, Performance evaluation of cross-digonal texture matrix method analysis, Pattern Recognition, Vol. 34, N ${ }^{\circ}$ 1, pp 171-120, 2001.

[12] D. Blostein and N. Ahuja, Sahape from texture: Integration texture-ellement axtraction and surface estimation. IEEE Transaction on Pattern Analysis and Machine Intelegence, Vol. 11, Nº1, pp. 1233-1251, 1989.

[13] M.L. Cormer and E.J. Delp, Segmentation of textured images using a multiresolution Gaussian autoregressive model. IEEE Transaction on Image Processing, Vol. 8, N³, pp. 408-420, 1999.

[14] G.M. Haley and B.S. Manjunath, Rotation-invaroiant texture classification using a complete space-frequency model. IEEE Transaction on Image Processing, Vol. 8, N² 2, pp. 255-269, 1999.

[15] T. Carron, Segmentation d'images couleur dans la base Teinte-Luminence-Saturation : approche numérique et symbolique. Thèse de doctorat, Université de Savoie, décembre 1995.

[16] M. K. Hu., Visual pattern recognition by moment invariants, IRE Trans. On information Theory, IT-8: 179-187, 1962.

[17] M. Sonka, V. Hlavac, and R. Boyle, Image Processing, Analysis and Machine Vision, PWS Publishing, $2^{\text {sd }}$ edition, 1999.

[18] A.C. Bovik, M. Clark and W.S. Geisler, Multichannel Texture Analysis Using Localized Spatial Filters, IEEE Transactions on Pattern Analysis and Machine Intelligence, Vol. 12, № 1, pp. 55-73, 1990.

[19] S.J. Sangwine et R.E.N. Horne, Te colour Image Processing HandBook. $1^{\text {st }}$ ed.. London : Chapman \& Hall, p. $440,1998$.

[20] Y.I. Ohta, T. Kanade, and T. Sakai, Color information for region segmentation. Computer Vision, Graphics, and Image Processing, 13:222-241, 1980.

[21] C. Pohl, J.L. Van Genderen, "Multisensor image fusion in remote sensing: concepts, methods and application", International Journal of Remote Sensing, vol. 99, no. 5, pp. 823-854, 1998.

[22] M. Costantini, A. Farina, F. Zirilli, "The fusion of different resolution SAR images", Proceedings of the IEEE, vol. 85, no. 1, pp. 139-146.

[23] W.J. Carper, T.M. Lillesand, R.W. Kiefer, "The use of intensity-hue-saturation transformations for merging SPOT panchromatic and multispectral image data", Photogrammetric Engineering and Remote Sensing, vol. 56, n. 4, pp. 459-467, 1990.

[24] B.S. Manjunath and W.Y. Ma, Texture features for browsing and retrieval of image data, IEEE Trans-actions on Pattern Analysis and Machine Intelligence, vol. $18 \mathrm{n}^{\circ} .8$, pp. $837\{842,1996$.

[25] J.B. MACQUEEN, Some Methods for classification and Analysis of Multivariate Observations, Proceedings of 5-th Berkeley Symposium on Mathematical Statistics and Probability, vol. 1, pp. 281-297, 1967. 